\title{
Datos sonificados para la improvisación visual
}

\author{
Sonificated data for visual improvisation
}

\author{
Bruno Perelli Soto \\ Universidad de Chile, Chile. \\ bperelli@uchile.cl \\ Eduardo Hamuy Pinto \\ Universidad de Chile, Chile. \\ ehamuy@uchile.cl
}

\author{
Rolando Cori Traverso \\ Universidad de Chile, Chile. \\ rcori@uchile.cl
}

\begin{abstract}
Citygram develops interactive environmental sensing through the capture of noise pollution in New York. Citygram data are received and reinterpreted in different parts of the world. a play based on musical and visual interpretation through improvisation develops, establishes a dialogue between performers and data. The work focuses on visualization, understood as artistic interpretation through images generated by devices programmed and manipulated in real time. The process combines conceptualization, plus integration of streaming technologies, software and similar visual programming compositional techniques. The result are three performances.
\end{abstract}

Keywords: Sonificación, Visualización, Improvisación, OSC.

\section{Introducción}

Sensing Urban Spaces Sound Map Citygram es un proyecto desarrollado por la New York University (NYU), con la colaboración de CalArts, NYC DEP, NYU CUSP, y NYU ITP. Desarrolla la detección interactiva del medio ambiente a través de la captura, cartografía, y exploración de las energías ambientales invisibles que convierten los espacios en lugares (Park, Miller, Shrestha, Lee, Turner y Marse, 2012; Citygram, 2016). Pretende favorecer una mayor conciencia sobre uno de los problemas que aquejan a las grandes ciudades, como es la contaminación acústica. La iniciativa, que data del año 2011, captura información ambiental sonora a través de dispositivos de tipo Remote Sensing Device (RSD) (Park et al., 2014) desplegados en distintos lugares de la ciudad de Nueva York, Estados Unidos (Citygram, 2016) la cual almacena en un servidor y transmite vía streaming. De ese modo permite la recuperación de datos de los $\mathrm{RSD}$, a los cuales se tiene acceso remoto en tiempo real a través de la interfaz de programación SuperCollider (SC) (2016).

El Proyecto U-Redes, titulado Red Interdisciplinaria de Arte (RIA), recibe a fines del 2014 la invitación a participar como parte de una iniciativa para que los datos de Citygram sean recibidos y reinterpretados en distintos lugares del Mundo. RIA apuesta, por medio de la improvisación artística a distancia, a establecer una plataforma de interacción entre artes y ciencias.

Para asumir el desafío de Citygram, RIA conforma un equipo compuesto por el grupo musical Tierra de Larry (patrocinado por el Departamento de Música y Sonología de la Facultad de Artes), un estudiante de postgrado en composición y dirección, y dos académicos del Departamento de Diseño, ambos departamentos de la Universidad de Chile.

Al inicio, se plantea generar una obra basada en la interpretación musical y visual a través de la improvisación, en respuesta al streaming de datos recibido, estableciendo un diálogo entre intérpretes y datos. Por una parte, se adscribe a la definición de Sonificación de Bly (en Worrall, 2012:312) dado que la interpretación musical representa datos multivariables a través de sonidos. Y por otra parte, se considera que existen

a. ... dos instancias de visualización, una visualización de índole mental o cognitiva, donde efectivamente se establece una imagen mental de un concepto abstracto, y por otra parte, tenemos una visualización de índole funcional, en la cual interactuamos con datos cualitativos o cuantitativos de carácter estadístico, que son procesados a través de un artefacto visual destinado a representar mediante imágenes ... fenómenos de otro carácter. (De la Sotta Lazzerini, Hamuy Pinto, \& Perelli Soto, 2013:550)

Con base en esta distinción, se determina que la Visualización, en el contexto del proyecto creativo Citygram, es la interpretación artística mediante imágenes generadas por artefactos programados y manipulados en tiempo real (improvisación) que hace el equipo de diseño, a partir de los mismo datos del streaming para la interacción con la sonificación.

\section{Concepto Creativo}

El arte en general se ha preocupado de lo caótico. A mediados del siglo $\mathrm{XX}$ surgen propuestas como las de Cage y Pollock, que toman el "ruido", sea este acústico o visual, como principio estético para mostrar el azar como sentido. 
El equipo de este proyecto de creación dialoga en un relato interactivo con la información paramétrica obtenida -la resonancia de una urbe lejana- Citygram. El bullicio del tráfico, personas, maquinarias, animales, viento y tantas otras fuentes, alimentan a los sensores que entregan como output un flujo de datos numéricos. Este flujo por sí solo es materia prima caótica para la vista o el oído, a la cual sujetos responden desde sus vivencias y experiencias como habitantes de otra urbe, transformándola en una improvisación artístico-musical-visual. El pie forzado de una interacción remota permite tomar conciencia de la vocación de corporeidad del género humano aún dentro de la coexistencia a veces rival y caótica de la ciudad. Esta mirada reflexiva de una telepresencia de lo humano (Merleau-Ponty, 2010), no busca instrumentalizar el dato numérico hacia un saber "objetivo positivo"[6] sino "el análisis en red [que] no revoluciona el objeto de estudio sino que cambia la mirada del investigador sobre él" (Merleau-Ponty, 2010:227). Contempla un concepto creativo que consiste en el proceso de búsqueda de un lenguaje expresivo coherente para representar el caos de la ciudad y los datos que genera, para develar un sentido de ellos a través de la sonificación y visualización.

Es necesario aclarar que el streaming recibido corresponde a parámetros del sonido -mediciones de variables sonorasmás no sonido expresado en forma digital. Por ejemplo, frecuencia promedio (centroide) de los sonidos capturados por los RSD, su energía, su variabilidad, etc. Estos datos son obtenidos a través del servidor de Citygram que, a su vez, tiene acceso a bancos de datos masivos (big data) acumulados por los sensores.

\section{Propósitos}

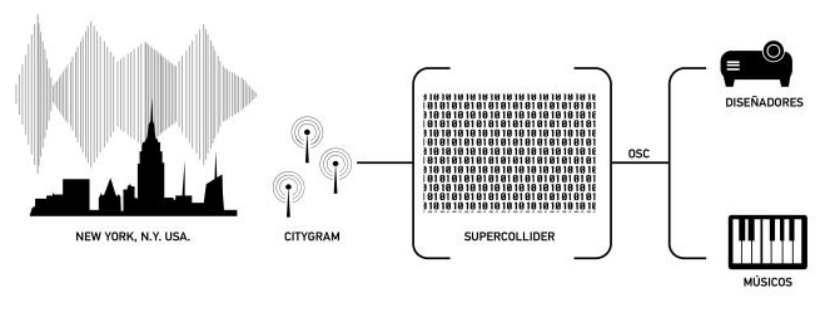

Figura 1: Esquema explicativo de cómo se hizo llegar la misma data en tiempo real y sincronizada a músicos y diseñadores.

Citygram, significa una etapa del proyecto RIA, que a través de superponer un flujo de datos remotos sobre ruido urbano a un espacio de improvisación visual y musical en red, explora la posibilidad de nuevas dimensiones de percepción del problema de la libertad y rivalidad como fenómenos propios del rumor de la civilización, en especial en el espacio urbano donde se manifiestan las artes y ciencias. Por la naturaleza improvisada de esta sonificación y visualización artística de los datos, el sentido que se pone en juego es el tacto, por lo que dimensiones mencionadas tienen un carácter bidireccional y reflexivo, pues hacen referencia al tocar y ser tocados por lo distante y lo cercano. Por esa razón las dimensiones, que podrán ser emotivas y cognitivas, serán de diversa índole y acento de acuerdo a la vivencia de los participantes, proyectando un sentido de intersubjetividad de una corporeidad extendida.

El presente trabajo se centra en la visualización, dejando la sonificación como un insumo para la mediación visual en diálogo con los intérpretes musicales y que podrá ser examinado con mayor detenimiento en futuros trabajos (Fig. 1). Los intérpretes visuales proponen primariamente, explorar la intervención de artefactos visuales en el espacio escénico, por medio de la improvisación e interacción con los datos numéricos en streaming, de forma tal de desarrollar un discurso visual respecto de lo que Citygram representa, manteniendo una coherencia y sincronía en comunión con la interpretación musical. Para improvisar en tiempo real distintas interpretaciones, se diseñan visuales que contemplan fotografías, video y figuras 3D geométricoabstractas que interactúan con datos numéricos.

De modo secundario requieren, generar un sistema de interfaces y artefactos para la manipulación e interacción del relato visual que surge de la improvisación grupal.

\section{Métodos}

Se describe ahora el proceso desarrollado por el equipo de visualización que permite cumplir con los propósitos declarados anteriormente. En primer lugar, como parte del proceso creativo, se lleva a cabo una exploración, en fases progresivas, que requiere primero establecer la conectividad técnica con el cuerpo de datos Citygram. Se comparte con el equipo de NYU las herramientas de software que permiten recibir en tiempo real el streaming de datos. De modo secundario requieren, generar un sistema de interfaces y artefactos para la manipulación e interacción del relato visual que surge de la improvisación grupal.

En segundo lugar, una vez establecida esa conectividad, la cual no siempre es estable y representa un desafío para los intérpretes ante eventuales disrupciones en la transmisión, se inicia un levantamiento de referentes visuales, tecnologías apropiadas y prototipos de artefactos. A partir de la revisión de referentes, como Memo Atken (http://www.memo.tv/amoeba-dance/), Nicholas Gould (https://youtu.be/qkk5wViJo-I), CSTNG-SHDWS para el video de Edison titulado Shadowtime (http://cstng-shdws.com) , Koyaanisqatsi el film the Godfrey Reggio (1982) y música de Philip Glass, se definen conceptos que orientan dos vertientes paralelas y complementarias para la preparación de material visual original. Por una parte se acopia un registro de escenas del transitar por el centro de la ciudad de Santiago, en video y fotografía. Se buscan imágenes de eventos representativos de efervescencia urbana, caos y orden en la ciudad, además de cámaras de control de tráfico (Unidad Operativa de Control de Tránsito de Santiago http://www.uoct.cl). Por otra, se explora la generación de imágenes con un común denominador, el uso de recursos tridimensionales y geometrías básicas. Esta segunda vertiente es elegida dado que al estar compuestas por vectores, responden a parámetros de construcción que dan la posibilidad de modificar sus superficies, puntos y colores. 


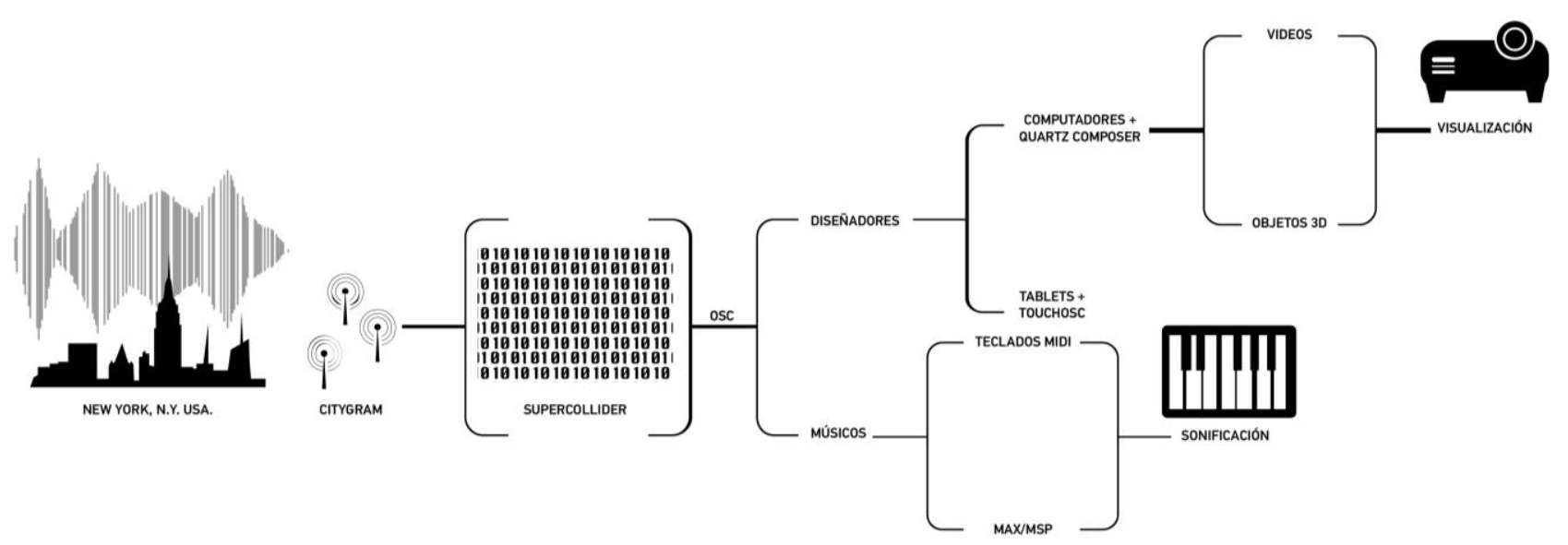

Figura 2: Esquema de distribución de data proveniente de Citygram y su uso como insumo tanto para los aspectos visuales como sonoros

A esto se suma que la aplicación de efectos propios de Quartz permite la alteración de su morfología según las iteraciones de datos entregados por SC, dando la impresión de que la figura respira, está viva y es responsiva al pulso del caos de la ciudad y al dato propuesto en el concepto creativo.

Tal como ya se ha señalado, en este proyecto SC es utilizado como puente entre los datos capturados y las salidas sonificadas por los músicos y visualizadas por los diseñadores. No obstante, si bien se ha logrado la conectividad gracias a SC, es mediante la utilización del protocolo Open Sound Control (OSC) que se logra alimentar de data a los artefactos de visualización, en desarrollo.

OSC es un protocolo de comunicación entre computadores, sintetizadores de audio y otros dispositivos con acceso a redes como son dispositivos móviles. Entrega los beneficios de los entornos de red para ser utilizdos en el mundo de los instrumentos musicales electrónicos (opensoundcontrol.org, 2011). Dado que es un criterio central en el diseño, mantener la coordinación entre data numérica leída, músicos y diseñadores a cargo de la visualización, para llevar a cabo la sincronización en la lectura de los datos, se utiliza OSC como protocolo de comunicación para alimentar Softwares de síntesis de audio y de programación visual: Quartz Composer, MAX MSP y TouchOSC (Fig. 2).

Quartz Composer es un software de programación visual en la que distintos componentes denominados Patches son unidos por nodos, similar a la lógica de MAX/MSP, Grasshopper o VVVV. Es un software gratuito que forma parte del entorno de desarrollo Xcode de Mac OS X y su principal función es la de procesar y representar datos gráficos. Al ser software abierto, existen diversos plugins gratuitos para expandir sus funciones permitiendo interactuar con una amplia cantidad de formatos multimediales, periféricos y otros sensores como Kinect y joysticks entre otros.
Si bien el programa cuenta con un módulo para la interpretación de data proveniente de OSC, las escasas actualizaciones han hecho que el módulo quede caduco. Sin embargo, Hexler, empresa desarrolladora de la app para dispositivos TouchOSC, cuenta con su propia versión del módulo OSC con la finalidad de que el usuario de Quartz pueda interactuar a través de una interfaz con dispositivos touchscreen móviles, como iPhone y iPad (Fig. 3). Podemos diseñar nuestras propias interfaces utilizando remotamente los parámetros visuales de Quartz y los parámetros numéricos entregados por SC. Estas interfaces son nuestras consolas de improvisación visual.

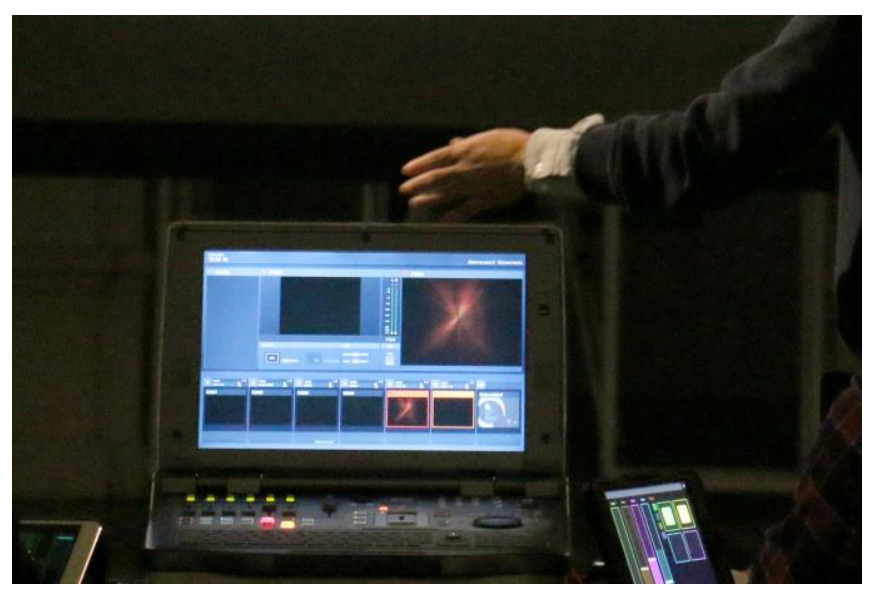

Figura 3: Visualizaciones realizadas en Quartz, gestionadas a través del sistema de producción de vídeo en directo Anycast y enlazadas con la interfaz TouchOSC que permite control durante la performace. Créditos: Prensa MAC.

Al ingresar en Quartz los datos de OSC provenientes de SC, el módulo comienza a registrar las entradas numéricas de datos provenientes del servidor de Citygram. Quartz utiliza estos datos para modificar parámetros en elementos como videos, alterando, la cantidad de fotogramas, su velocidad, la aplicación de filtros o los parámetros para geometrías 3D al modificar elementos como ubicación de puntos, cantidad de polígonos, entre otros. En síntesis, los visualizadores han 
configurado sus propios instrumentos y comienzan a trabajar en dimensión estética e interpretativa.

Durante un período de ocho meses se sostiene un proceso regular de ensayos con integrantes del grupo Tierra de Larry y quien ejecutará la dirección musical en escena. A partir de un mes antes de la primera presentación pública, se realizan ensayos y pruebas técnicas en el Museo de Arte Contemporáneo de la Universidad de Chile (MAC) donde participan presencialmente los equipos de sonificación y visualización. Como alternativa ante la eventualidad de intermitencia de acceso al streaming, se configuran interfaces y procedimientos de improvisación de visuales que permiten el manejo de parámetros de modo manual. En el proceso de ensayos en el MAC se incorpora un sistema de producción de video en directo Anycast, el cual permite mezclar y hacer transiciones entre dos canales de video, provenientes de cada uno de los intérpretes de visualización.

Unos días antes del estreno se hacen pruebas técnicas y unos ensayos con el grupo musical que actuará en red desde Córdoba, Argentina. Se evidencia la necesidad de enviar contenidos de video pregrabado a estos últimos, pues por limitaciones de ancho de banda no resulta estable la transmisión de datos, se decide dar prioridad a la transmisión de audio entre ambos países para resguardar la fluidez durante la performance.

\section{Resultados}

A partir del proceso de experimentación descrito, se realizan tres presentaciones que colocan en circulación la obra. La primera, es una interpretación en streaming en el marco de la red iberoamericana Anilla Cultural Latinoamérica Europa, de la que participa el MAC, y una colaboración con el Festival de Música Electroacústica Experimentalia, organizado por el Centro Cultural España Córdoba, Argentina, y que se realiza en septiembre de 2015. En esa oportunidad, las visuales mezcladas en tiempo real por los diseñadores, son un insumo para dos grupos de músicos: Tierra de Larry presente en el MAC y otro remoto, el grupo Red Ensamble que, desde Argentina, recibe y envía señal audiovisual. Ambos generan una improvisación paralela, construyendo colectivamente la obra. Tal como permite observar la imagen (Fig. 4), los intérpretes se distribuyen en semicírculo enfrentando el telón donde se proyectan la imagen proveniente de Córdoba junto a la proyección de las distintas interpretaciones visuales del cuerpo de datos. De este modo, los diferentes intérpretes mantienen contacto visual entre sí, las visuales y un director musical, quien se ubica al centro del escenario y mediante gestos similares a los de un conductor, administra entradas, duración, intensidad y carácter de las intervenciones musicales y visuales en ambos puntos de la red.

La segunda presentación se realiza en la Casa Central de la Universidad de Chile, en el marco del Foro de las Artes. En esta oportunidad, al igual que la siguiente, solo participan los intérpretes de visualización junto a Tierra de Larry. Un tercera presentación se realiza en el XII Festival Internacional de Música Contemporánea Darwin Vargas 2015 en Valparaíso. Se acuerda previamente un modo de iniciar la performance a partir de la exhibición concreta de los datos numéricos en la pantalla y un sonido muy básico que es modificado por el streaming. El otro esquema básico que se acuerda entre los intérpretes, es deambular grupalmente por zonas de menor y mayor intensidad sonora y visual, otorgando alternadamente protagonismo a estos medios. A través del contacto visual que se mantiene entre los intérpretes, se coordinan entradas, duración, intensidad y carácter de las intervenciones musicales y visuales, a lo largo de una pieza única que se concluye de modo coordinado. Cada presentación tiene una duración diferente, entre 45 y 30 minutos.

\section{Discusión}

El proceso creativo y de experimentación en este proyecto artístico, combina diferentes dimensiones y fases. Se inicia el proyecto con un período de reflexión y conceptualización en torno a la experiencia de habitar en la urbe enfrentados a una vorágine de estímulos que se presentan como un caos, un sin sentido. No obstante, se busca además de representar ese caos por medio de la sonificación y visualización, también transformar ese constante devenir en un discurso que toca a los intérpretes y a la audiencia. Cada presentación es única, resulta de la percepción de los intérpretes y la retroalimentación que se produce durante la performance, que ellos - al igual que lo que señalan algunos miembros de la audiencia- experimentan como una travesía por una serie de paisajes sonoros y visuales. Ese discurso surge desde la sensibilidad individual y grupal de los intérpretes, en su interacción en el lugar compartido del escenario físico, el mediado por las redes y la experiencia de lo urbano. Todo esto, dado en el momento único e irrepetible de la improvisación. 


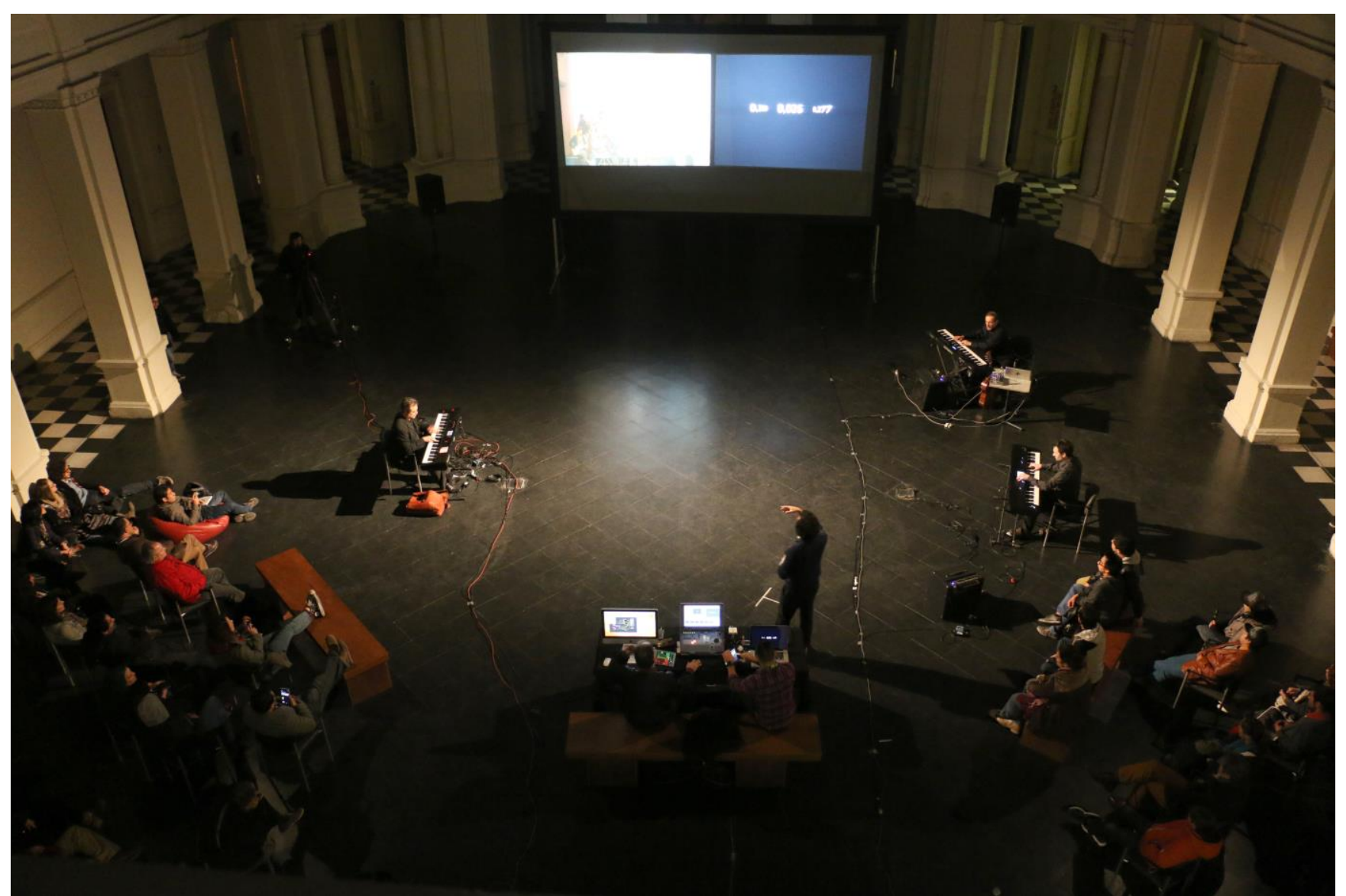

Figura 4: Presentación del día 25 de septiembre de 2015, Museo de Arte Contemporáneo (MAC). Se aprecia al centro arriba, la proyección de visualizaciones (zona izquierda) en conjunto a la transmisión proveniente de Córdoba (zona derecha). La agrupación Tierra de Larry (ambos lados, al medio), el Director, Jorge Pacheco, junto con el equipo de diseño de visualizaciones (abajo, al centro).

Créditos: Prensa MAC.

Para lograr ese resultado se ocupan recursos como el rol de la conducción musical que coordina acuerdos estéticos y sintácticos que se han construido a partir de un proceso de meses de exploración y ensayos. También se desarrolla en ese tiempo, la indagación grupal de posibilidades de integración de tecnologías de streaming y visualización con propósitos de generar una performance en tiempo real. El resultado es una combinación de lo digital y análogo en la interpretación, una coordinación técnica y rapport empático entre los intérpretes.

\section{Agradecimientos}

Queremos agradecer a Lars Guargaard (lars@graugaardmusic.dk) quien estableció la coordinación con el equipo de Citygram en NYU. A Alessandra Burotto coordinadora del equipo del Proyecto Anilla Cultural MAC por su excelente apoyo. A Jorge Pacheco por su participación durante todo el proceso creativo y su rol de director musical. Al Grupo "Tierra de Larry" (Edgardo Cantón, Leonardo Cendoyya y Rolando Cori) por su generosa invitación a participar en el proyecto; y a la Vicerrectoría de Investigación y Desarrollo de la Universidad de Chile que a través del fondo U-Redes, auspicia a la Red Interdisciplinaria de Arte.

\section{Referencias}

Citygram (2016) http://citygram.smusic.nyu.edu NYU, Department of Music, Park, Tae Hong, Project lead. http://cds.nyu.edu/projects/citygram-sound/ Consultado 11/7/16.

De la Sotta Lazzerini, P., Hamuy Pinto, E., \& Perelli Soto, B. (2013). Diseño de Aplicación en Base a Modelo de Evaluación del Proceso de Enseñanza Aprendizaje en El Taller Introducción Metodología Resultados / A Design Studio Course Assessment Model Applied. In SIGraDi 2013. Proceedings of the 17th Conference of the Iberoamerican Society of Digital Graphics: Knowledge-based Design (pp. 547-550). Valparaíso, Chile: USM Editorial. http://doi.org/10.5151/despro-sigradi2013-0106

Grandjean, M. (2014). La connaissance est un réaseau, Perspective sur l'organisation archivistique et encyclopédique. http://www.martingranjean.ch

Merleau-Ponty, M. (2010)Lo visible y lo invisible, traducción Estela Consigli y Bernard Capdevielle, Nueva Visión, Buenos Aires.

OSC Open Sound Control (2011). Recuperado de http://opensoundcontrol.org

Park, T. H., Miller, B., Shrestha, A., Lee, S., Turner, J., \& Marse, A. (2012). Citygram One: Visualizing Urban Acoustic Ecology. In Digital Humanities 2012.

Park, T. H., Musick, M., Turner, J., Mydlarz, C., Lee, J. H., You, J. y DuBois, L. (2014) Citygram One: One Year Later ... 
SIGraDi 2016, XX Congreso de la Sociedad Ibero-americana de Gráfica Digital

9-11, November, 2016 - Buenos Aires, Argentina

Proceedings ICMC|SMC|2014, 14-20 September 2014, Athens, Greece.

SuperCollider (2016) McCartney, James. 1996 - 2016. General Public License http://supercollider.github.io Consultado 11/7/16.

Worrall, D. (2012). An introduction to data sonification. En Dean, R. T. (ed.) The Oxford Handbook of Computer Music. The Oxford Handbook of Computer Music, 1-624. http://doi.org/10.1093/oxfordhb/9780199792030.001.0001 